\title{
A Case of Very Early Stent Restenosis Following DES Implantation
}

\author{
Shravan Kumar Ch. ${ }^{1} \quad$ Naveen Kumar M. ${ }^{1} \quad$ Sudhakar Kanumuri ${ }^{1}$ \\ ${ }^{1}$ Department of Cardiology, Nizams Institute of Medical Sciences, \\ Hyderabad, Telangana, India \\ Address for correspondence Shravan Kumar Ch., DCH, DNB, DM, \\ Department of Cardiology, Nizams Institute of Medical Sciences, \\ Hyderabad, TS 500 082, Telangana, India \\ (e-mail: shravankch@gmail.com).
}

Ind J Car Dis Wom 2019;4:200-203

\begin{abstract}
Keywords

- in-stent restenosis

- optical coherence tomography

- neointimal hyperplasia

Intracoronary in-stent restenosis (ISR) is more common with bare metal stents. With the introduction of drug-eluting stents (DESs), the incidence of ISR has markedly decreased. Here, we report a case of unusual very early stent restenosis in a patient who presented with unstable angina 15 days after percutaneous coronary intervention with sirolimus-eluting DES. Optical coherence tomography (OCT) was done to know the pathophysiology of the very early stent stenosis and the possibilities of the rare findings of this OCT are discussed.
\end{abstract}

\section{Introduction}

Intracoronary in-stent restenosis (ISR) is a gradual renarrowing of the stented segment of the vessel. Normal occurrence is 3-12 months after angioplasty. It is more common with bare metal stents. With the introduction of drug-eluting stents (DESs), the incidence of ISR has markedly decreased. Here, we report a case of unusual very early stent restenosis of DES in a coronary artery disease (CAD) patient.

\section{Case Report}

A 35-year-old man who had effort angina for 3 months was diagnosed with chronic stable angina, with normal left ventricle (LV) function, and underwent coronary angiogram, which was suggestive of single-vessel disease (mid-left anterior descending artery [LAD] 80\% stenosis). The patient was referred to our hospital for intervention. He underwent percutaneous coronary intervention (PCI) to mid-LAD with $2.5 \times 32 \mathrm{~mm}$ sirolimus-eluting DES. Post procedure, he was on dual antiplatelet therapy (DAPT) and discharged. After 15 days, he presented with complain of typical angina at the rest of two days duration to our outpatient department (OPD). He was evaluated, electrocardiogram (ECG) showed no significant ST-T changes, troponin T was negative, and the LV function was normal. As there was no evidence of stent thrombosis, we did stress thallium, which showed inducible ischemia in LAD territory. He was admitted with provisional diagnosis as unstable angina and treated accordingly.
The patient underwent a coronary angiogram, which revealed mid-LAD in-stent focal $90 \%$ stenosis (-Fig. 1). There was no angiographic evidence of thrombus. To know the pathophysiology for such very early stent restenosis in DES, optical coherence tomography (OCT) guidance PCI was planned. OCT imaging is done after balloon dilatation in view of tight stenosis. There was no evidence of thrombus; instead, rapidly growing granulation tissue most likely fibrofatty layer, mostly fat tissue near the stent struts (hypodense areas) with good stent expansion was seen ( - Fig. 2). In addition, hypodense tissues were seen outside the stent also, which may represent the fast-growing granulation tissue ( - Fig. 3). Considering very early aggressive neointimal hyperplasia, sequential balloon dilatation was done, followed by an everolimus-eluting stent (xience prime) of 2.5 $\times 18 \mathrm{~mm}$ stent implanted. Post implantation check injection thrombolysis in myocardial infarction (TIMI) 3 flow was

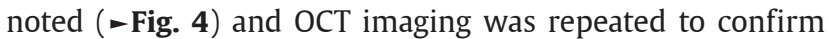
the good expansion of the stent and to see for any edge dissections or other complications ( - Fig. 5). Post procedure, the patient improved symptomatically and was discharged in stable condition on DAPT. In 6 months follow-up there was no recurrence of symptoms.

\section{Discussion}

Intracoronary ISR is commonly seen 3 to 6 months after bare metal stents; with DES, the incidence of ISR has decreased. The most common presentation is unstable angina, rarely

(C)2019 Women in Cardiology and Related Sciences
License terms

()(1) $\Theta \circledast$ 


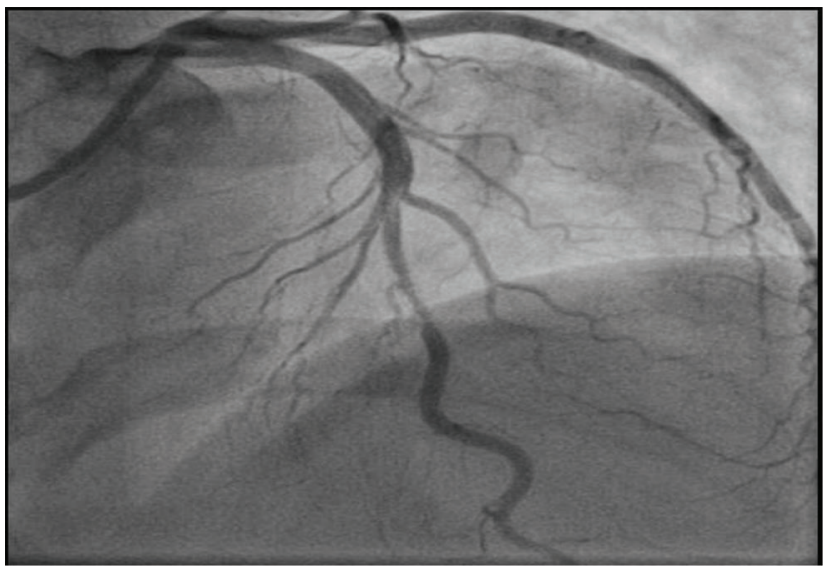

Fig. 1 Check angiogram AP cranial view showing mid-LAD stenosis. AP, antero posterior.

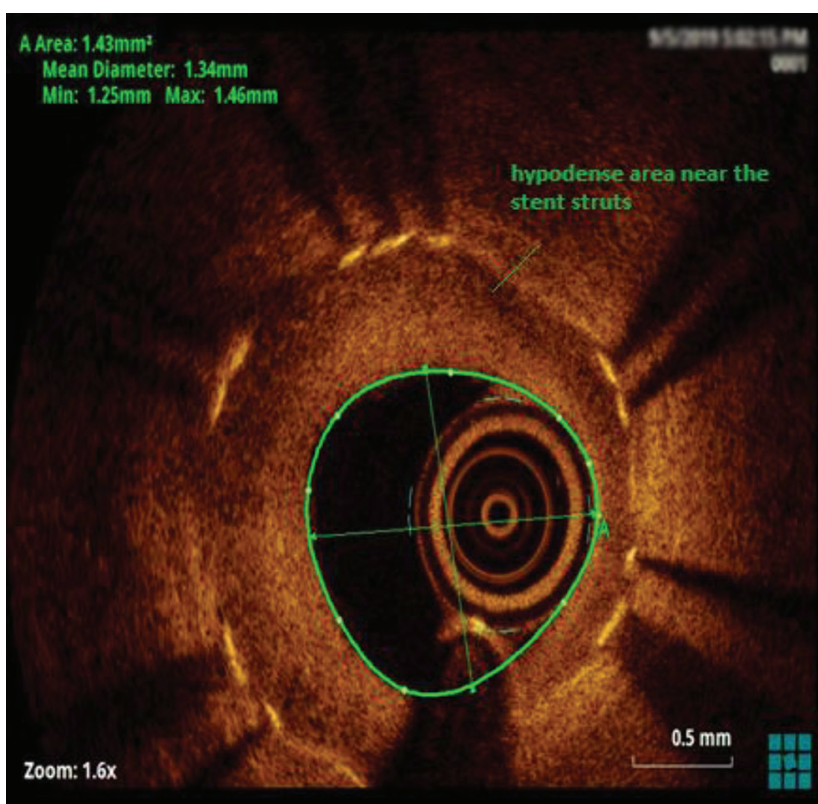

Fig. 2 Optical coherence tomography suggestive of stent in-stent restenosis with layered neointima, hypodense areas near the stent struts.

as acute myocardial infarction (MI) in $10 \%$ of cases. ${ }^{1}$ Among first-generation DES (sirolimus or paclitaxel) ISR is 13 to $16 \%$ at 5 years. ${ }^{2,3}$ Among second-generation DES (everolimus-eluting and zotarolimus-eluting stents), 5 to $6.3 \%$ ISR is noted. ${ }^{4}$ Many factors like drug component of stents, hypersensitivity, stent under expansion, and barotrauma outside the stented segment will influence the subsequent development of ISR. ${ }^{5}$

Angiographically, ISR can be of four patterns-focal ( $<10 \mathrm{~mm}$ in length) lesion, ISR $>10 \mathrm{~mm}$ within the stent, ISR $>10 \mathrm{~mm}$ extending outside the stent, and totally occluded stent. ${ }^{6}$ The incidence decreases and revascularization requirement increases from pattern one to four. ISR risk is more in target vessel with lesion characteristics like morphology of lesion (type c, more risk of ISR), ${ }^{7}$ preintervention plaque burden, ostial location, stent length, longer lesion, residual plaque burden after implantation, post stent longitudinal straightening effect, minimum lumen diameter

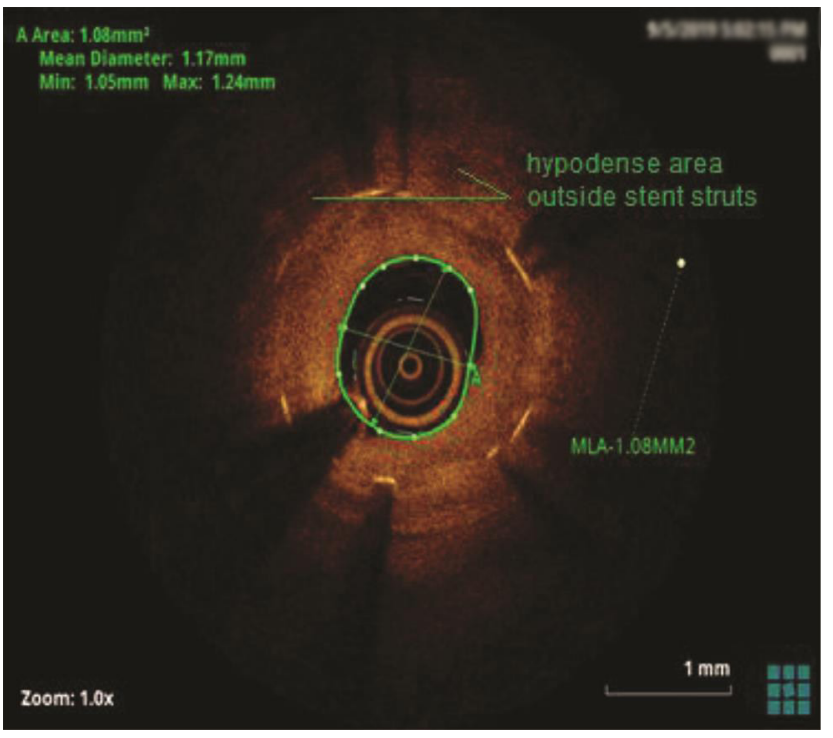

Fig. 3 Hypodense area outside the stent struts.

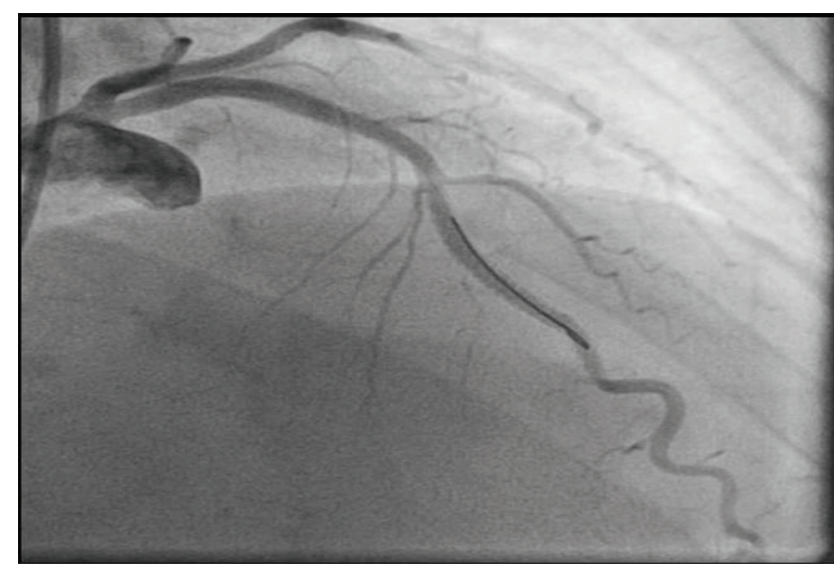

Fig. 4 Post PCI RAO cranial view suggestive of TIMI 3 flow. PCI, percutaneous coronary intervention; RAO, right anterior oblique.

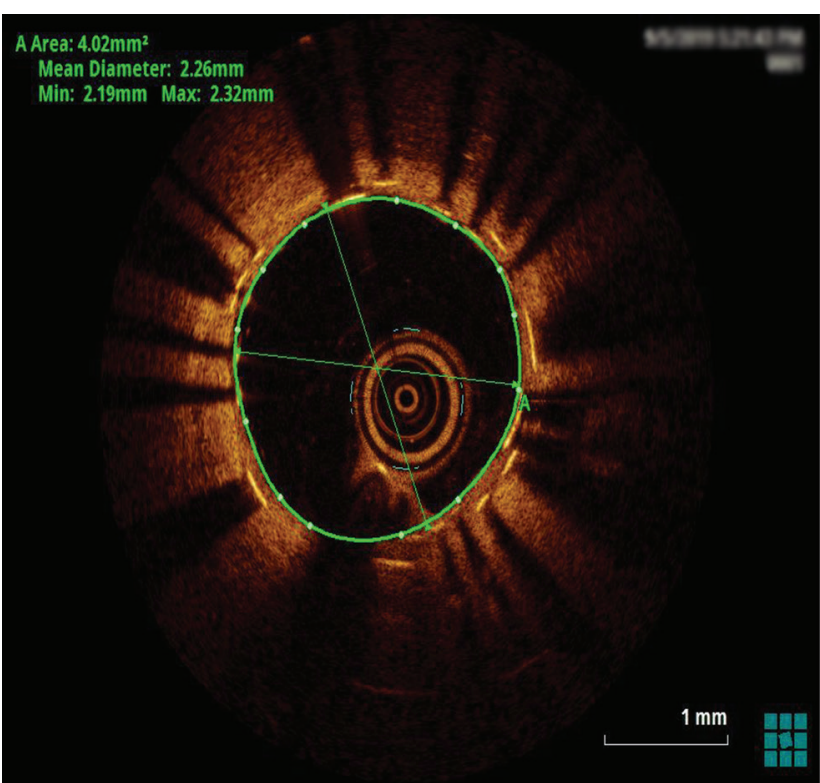

Fig. 5 Post $\mathrm{PCl}$ optical coherence tomography image suggestive of good stent expansion. 
$<3 \mathrm{~mm}$, late lumen loss, and minimum stent area. ${ }^{8}$ Clinical factors play a major role in target vessel revascularization in bare metallic stent (BMS) than in DES. Predictors of ISR in the second generation DES include type 2 diabetes, ostial lesions, venous graft $\mathrm{PCI}$, and stent under expansion.

Two important features of this case were very early restenosis and plaque morphology of ISR on OCT. As far as our knowledge goes, so early ISR in DES was not reported till now. There were two case reports of ISR after 2 months in previously acute stent thrombosis cases by Corrando Tamburino. ${ }^{9}$

After stent implantation, there is initially migration of inflammatory cells, initiation of endothelialization, deposition of fibrin, then smooth muscle cell proliferation, development of neointima rich in collagen, and proteoglycans, which give optical signals that are similar to fibrous tissue during imaging. After $\mathrm{PCl}$ within the first 2 years, neointimal proliferation is the main cause of ISR, then afterward, neointimal atherosclerosis mainly of fibroatheroma. ${ }^{10,11}$

OCT will be helpful in a comprehensive understanding of the restenosis distribution and it will help understand the composition of the neointima. There are three morphological patterns of neointima have been identified on OCT.

Homogenous neointima: Normal in-stent proliferative response. High backscattering and low attenuation characteristics due to the high content of smooth muscle cells and collagen fibers.

Layered neointima: High backscattering superficial layer and low backscattering deeper layer.

Heterogenous neointima: Speckled pattern with areas of high and low backscattering distributed throughout the tissue. Causes of low backscattering include fibrin accumulation, organized thrombus, and inflammation. ${ }^{6,12}$

In the present case, significant hypodense areas were seen near the stent struts and even outside the stent. Hypodense areas on OCT represents the fatty tissue, which is likely to present though out the thickness or at the luminal surface but not so periphery of plaque. Usually fibrofatty lesions show mixed density, not like this hypodensity in the periphery of the plaque. The same hypodensity outside the stent also cannot be explained by fat deposition. So, we are proposing that that rapidly proliferating granulation tissue was responsible for these hypodensities both inside and outside the stent (may be activated macrophages from media during stent implantation), which requires histopathological confirmation.

Main treatment options for ISR include either drugcoated balloon or stents for target vessel revascularization (TLR) irrespective of the previous stent, whether BMS or DES. Placement of a newer generation DES, and in particular an everolimus-eluting stent, is the preferred treatment for patients with ISR. ${ }^{13}$ In a meta-analysis comparing everolimus DES with DEB, combined clinical outcome of MI, cardiac death, TLR is shown to be lower with everolimus DES than DEB (12.3 vs. $20.1 \%$; hazard ratio 0.57 , $95 \%$ 234-0.96).
In our present case, we have an initial suspicion of stent thrombosis than ISR as he is presented early with an acute coronary syndrome. However, to our surprise during intraluminal imaging with OCT characteristics like neointimal hyperplasia, granulation tissue, a hypodense area near stent struts, and good stent expansion were noted. We used the everolimus-eluting stent, which is one of the recommended treatment options by which the patient improved. Here we further emphasize the role of image-guided routine PCI to know the lesion characteristics and stent expansion and edge dissections.

\section{Conclusion}

The uniqueness of this case is so early in-stent (DES) restenosis confirmed by OCT along with the abnormal plaque morphology (hypodense areas near and outside the stent struts) in addition to classical neointimal hyperplasia. OCT is an important image to know the pathophysiology and mode of treatment in early in-stent stenosis.

\section{Conflict of Interest}

None.

\section{References}

1 Mishkel GJ, Moore AL, Markwell S, Shelton MC, Shelton ME. Long-term outcomes after management of restenosis or thrombosis of drug-eluting stents. J Am Coll Cardiol 2007;49(2):181-184

2 Kimura T, Morimoto T, Nakagawa Y, et al. j-Cypher Registry Investigators. Very late stent thrombosis and late target lesion revascularization after sirolimus-eluting stent implantation: five-year outcome of the j-Cypher Registry. Circulation 2012;125(4):584-591

3 Räber L, Wohlwend L, Wigger M, et al. Five-year clinical and angiographic outcomes of a randomized comparison of sirolimus-eluting and paclitaxel-eluting stents: results of the Sirolimus-Eluting Versus Paclitaxel-Eluting Stents for Coronary Revascularization LATE trial. Circulation 2011;123(24):28192828, 6, 2828

4 Piccolo R, Stefanini GG, Franzone A, et al. Safety and efficacy of resolute zotarolimus-eluting stents compared with everolimus-eluting stents: a meta-analysis. Circ Cardiovasc Interv 2015;8(4):e002223

5 Dangas GD, Claessen BE, Caixeta A, Sanidas EA, Mintz GS, Mehran R. In-stent restenosis in the drug-eluting stent era. J Am Coll Cardiol 2010;56(23):1897-1907

6 Mehran R, Dangas G, Abizaid AS, et al. Angiographic patterns of in-stent restenosis: classification and implications for long-term outcome. Circulation 1999;100(18):1872-1878

7 Kastrati A, Schömig A, Elezi S, et al. Prognostic value of the modified American college of Cardiology/American heart association stenosis morphology classification for long-term angiographic and clinical outcome after coronary stent placement. Circulation 1999;100(12):1285-1290

8 Mauri L, O'Malley AJ, Cutlip DE, et al. Effects of stent length and lesion length on coronary restenosis. Am J Cardiol 2004;93(11):1340-1346,A5

9 Tamburino C, Ussia GP, Zimarino M, Galassi AR, De Caterina R. Early restenosis after drug-eluting stent implantation: a putative role for platelet activation. Can J Cardiol 2007;23(1):57-59 
10 Buja LM. Vascular responses to percutaneous coronary intervention with bare-metal stents and drug-eluting stents: a perspective based on insights from pathological and clinical studies. J Am Coll Cardiol 2011;57(11):1323-1326

11 Nakazawa G, Otsuka F, Nakano M, et al. The pathology of neoatherosclerosis in human coronary implants bare-metal and drug-eluting stents. J Am Coll Cardiol 2011;57(11):1314-1322
12 Nakano M, Vorpahl M, Otsuka F, et al. Ex vivo assessment of vascular response to coronary stents by optical frequency domain imaging. JACC Cardiovasc Imaging 2012;5(1):71-82

13 Alfonso F, Pérez-Vizcayno MJ, Cuesta J, Rivero F, García-Touchard A, García Del Blanco B; RIBS IV Investigators. Paclitaxel-eluting balloons or everolimus-eluting stents for in-stent restenosis. JACC Cardiovasc Interv 2018;11(5):505-506 